\title{
The Realization and Working Conditions of Memristor Based on Multisim*
}

\author{
Dehua Song ${ }^{1}$, Xiang Ren ${ }^{1}$, Mengfei Lv ${ }^{1}$, Mengmeng Li ${ }^{1}$, Haiyang Zhou', Yunxiao Zu \\ ${ }^{1}$ School of Information and Telecommunication Engineering, Beijing University of Posts and Telecommunications, Beijing, China; \\ ${ }^{2}$ School of Electronic Engineering, Beijing University of Posts and Telecommunications, Beijing, China. \\ Email: zuyx@bupt.edu.cn
}

Received July 2013

\begin{abstract}
An equivalent circuit is realized using Multisim software by transforming a kind of circuit element according to Mapping principle and circuit theory. The effects of every parameter on the equivalent circuit are analyzed and the working conditions of the equivalent circuit are concluded by simulation.
\end{abstract}

Keywords: Memristor; Multisim; Equivalent Circuit; Realization; Working Conditions

\section{Introduction}

Four researchers from HP laboratory successfully manufactured memristor based on the metal and metal oxide in Nano-scale in 2008 and found the mathematical model of memristor [1]. The memristor was being focused since then [2-11]. However, analysing the circuit containing memristor is very difficult because the voltage-current relation (VCR) of memristor is a multivalued function. Though researchers usually use a linearization approach to analyse the circuit, it is also difficult and inaccurate. A mutator converting other circuit components into memristors is built in this paper according to the mapping principle and circuit theory based on Multisim in order to analyse circuit containing memristor easily and accurately.

Memristor is an element which provides a functional relation between flux linkage $\psi$ and charge $q$, and the basic model is as follows.

$$
\mathrm{d} \psi=M \mathrm{~d} q
$$

where $\mathrm{M}$ is the memristance of the memristor.

\section{Circuit of Mutator}

Mutator converts VCR of an element into $\Psi$-q relation to simulate memristor. The circuit of mutator is shown in Figure 1.

\section{The Realization of Memristor Based on Multisim}

Assigning $h_{m}=0.4, k_{m}=1000, \mathrm{~L}=1 \mathrm{mH}, \mathrm{C}=1 \mathrm{mF}, \mathrm{R}=$

*Project supported by the research innovation fund for college students of Beijing University of posts and telecommunications.
$1 \Omega, \mathrm{I}=1 \mathrm{~A}$. The $u$ - $t$ relation of memristor is shown in Figure 2 by simulation in Multisim. The longitudinal axis scale is $500 \mathrm{~V} / \mathrm{Div}$. It can be seen that the curve is a straight line paralleled with axis $t$, so the memristor is equivalent to a linear resistor.

Memristor with fixed $\mathrm{M}$ is converted by linear resistor, so no research value. Only $\mathrm{M}$ is variable can memristor has memory function, so a nonlinear component as the converted component is necessary. The diode can be converted a memristor with variable $\mathrm{M}$. Because the VCR cannot be shown directly in Multisim, a $1 \Omega$ resistor is in series in the circuit. In this way, the current of the circuit can be detected because the voltage and the current of the $1 \Omega$ resistor are numerically equal. The channel A of the oscilloscope is used to detect the voltage of memristor while the channel B is used to detect the voltage of $1 \Omega$ resistor, then the VCR can be obtained using the $\mathrm{A} / \mathrm{B}$ mode.

The VCR of the equivalent memristor can be obtained when the sine alternating current $i=\sin (2 \pi f \mathrm{t}) \mathrm{A}, f=1$ $\mathrm{kHz}$ is added on the circuit and let $h_{m}=0.4, k_{m}=1000, \mathrm{~L}$ $=1 \mathrm{mH}, \mathrm{C}=1 \mathrm{mF}$. The $u$ - $t$ relation is shown in Figure 3 . The horizontal axis scale is 500 us/Div and the longitudinal axis scale is $500 \mathrm{mV} / \mathrm{Div}$. The VCR of memristor is shown in Figure 4. Both the horizontal axis scale and the longitudinal axis scale are $500 \mathrm{mV} / \mathrm{Div}$. It can be seen that the VCR of the equivalent memristor is an inclined "8" and very close to the VCR in literature [2]. So the circuit can be as the equivalent memristor with little error.

The circuit of converting the diode into memristor in 


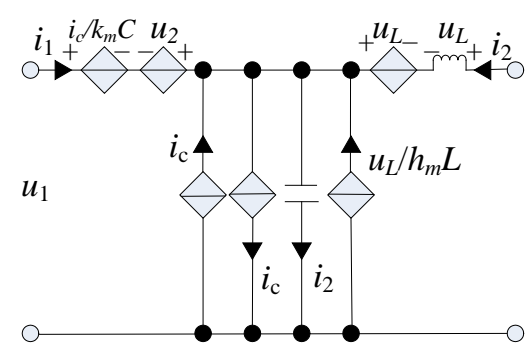

Figure 1. Circuit of mutator.

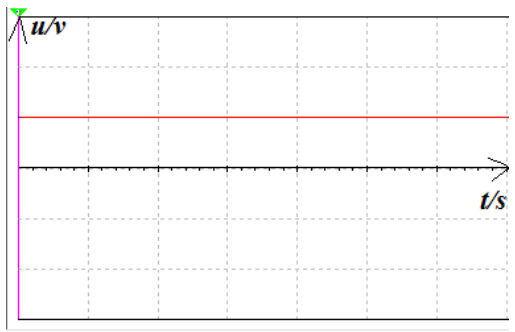

Figure 2. The $u$-t relation.

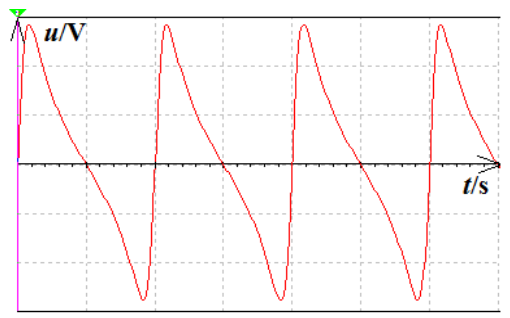

Figure 3. The $u$-t relation.

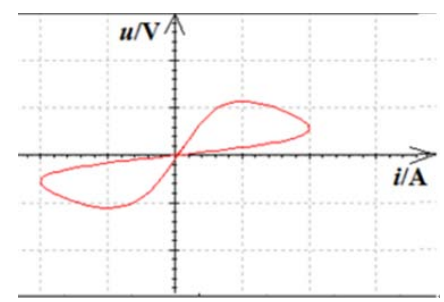

Figure 4. The VCR of the equivalent memristor.

Multisim is shown in Figure 5.

\section{The Working Conditions of the Equivalent Circuit}

\subsection{The Influences of $h_{m}$ and $k_{m}$}

Put

$$
\left\{\begin{array}{l}
u=k_{m} \psi \\
i=h_{m} q
\end{array}\right.
$$

into the VCR of the diode $i=I_{\mathrm{s}} \mathrm{e}^{\frac{u}{U_{t}}}$, Equation (3) can be gotten.

$$
h_{m} q=I_{\mathrm{s}} \mathrm{e}^{\frac{k_{m} \psi}{U_{t}}}
$$

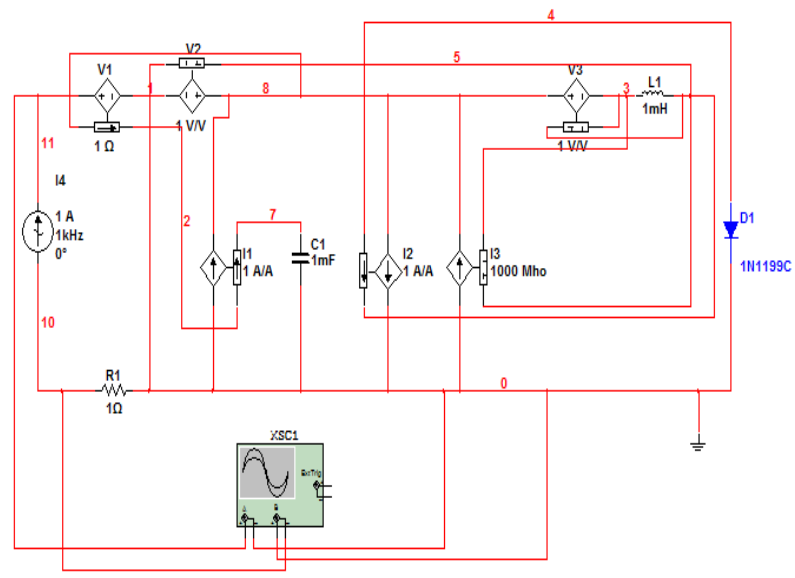

Figure 5. The simulation circuit in Multisim.

Simplifying Equation (3)

$$
\frac{k_{m} \psi}{U_{\mathrm{t}}}=\ln \frac{h_{m} q}{I_{\mathrm{s}}}
$$

Making differential on $\mathrm{t}$ in both sides of Equation (4).

$$
\frac{k_{m}}{U_{\mathrm{t}}} \frac{\mathrm{d} \psi}{\mathrm{d} t}=\frac{I_{\mathrm{s}}}{h_{m} q} \frac{h_{m}}{I_{\mathrm{s}}} \frac{\mathrm{d} q}{\mathrm{~d} t}
$$

Put $u=\frac{\mathrm{d} \psi}{\mathrm{d} t}, i=\frac{\mathrm{d} q}{\mathrm{~d} t}$ into Equation(4).

$$
\frac{k_{m}}{U_{t}} u=\frac{1}{q} i
$$

Put $q=\int_{0}^{t} i \mathrm{~d} t$ into Equation (6).

$$
u=\frac{U_{\mathrm{t}}}{k_{m}} \frac{i}{\int_{-\infty}^{t} i \mathrm{~d} t}
$$

Analyzing Equation(7) and can found that with the same current, the bigger $h_{m}$, the smaller the voltage and the resistance of the equivalent circuit. Assigning $I_{s}=0.1$ $\mathrm{uA}, \mathrm{U}_{\mathrm{t}}=26 \mathrm{mV}, i=\sin (2 \pi f \mathrm{t}) \mathrm{A}, f=1 \mathrm{kHz}, h_{m}=1$, then the $u-t$ relation for different $k_{m}$ in MATLAB simulation is shown in Figure 6.

It can be seen from Figure 6 that the voltage peak varies with different $k_{m}$. The bigger $k_{m}$, the smaller the voltage peak will be. Although the changing rate of the voltage is variable, the $u$ - $t$ relations for different $k_{m}$ have not intersected except the origin. Because the value of the current source is the same at same moment, the bigger the voltage, the bigger the resistance of the equivalent circuit, and at different moment the resistance is bigger for smaller $k_{m}$.

The simulation waveform is the same with that of theoretical analysis. For the same current source, the smaller $k_{m}$, the bigger the voltage peak. Assigning $\mathrm{L}=1 \mathrm{mH}, \mathrm{C}=$ $1 \mathrm{mF}$ and $i=\sin (2 \pi f \mathrm{t}) \mathrm{A}, f=1 \mathrm{kHz}$. The VCR gradually 


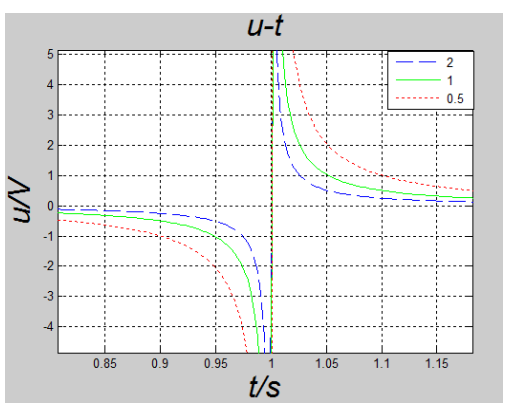

Figure 6. The $u$-t relation for different $\boldsymbol{k}_{\boldsymbol{m}}$.

approaches to a straight line and closes to i axis when $k_{m}$ increasing, while the shape of the $u$ - $t$ curve does not change but the voltage peak decreasing. Assigning $k_{m}=$ $5000, h_{m}=0.4$, and the VCR of the equivalent circuit is shown in Figure 7, in which both the horizontal axis scale and the longitudinal axis scale are $500 \mathrm{mV} / \mathrm{Div}$. Increasing $k_{m}$ until the oscilloscope can not display, however the shape of VCR still remains the same. Decreasing $k_{m}$, the VCR of the equivalent circuit gradually approaches to a straight line and closes to u axis. When $k_{m}$ changes very little, the shape of $u$ - $t$ curve does not change but the voltage peak increasing. When $k_{m}$ is smaller than $10^{-7}$, the $u$ - $t$ curve of the equivalent circuit approaches to the sinusoid and shows the properties of linear resistor rather than memristor with changing memrisistance. Assigning $k_{m}=10^{-7}, h_{m}=0.4$, and the $u-t$ relation of the equivalent circuit is shown in Figure 8, and in which the horizontal axis scale is $500 \mathrm{us} / \mathrm{Div}$ and the longitudinal axis scale is $500 \mathrm{MV} / \mathrm{Div}$. It can be seen that the curve is very close to sinusoid. Because some elements of the equivalent circuit are active elements, the circuit takes some time to get stable, the stability of the circuit will not be changed when changing $k_{m}$, that is the VCR curves will never overlap. In a word, when $h_{m}=0.4$, the error of the circuit is small if $k_{m}$ is between 10 and $10^{4}$.

The impact of $h_{m}$ on the equivalent circuit is different from that of $k_{m}$. For the same current source, the time the voltage in the $u$ - $t$ curve reaching zero is the same for different $h_{m}$, and that is right for the voltage reaching the peak. $h_{m}$ has no impact on the time the voltage reaching the peak. The simulation waveform in Multisim is different from that of the theoretic analysis. For the same current source, the bigger $h_{m}$, the bigger the voltage peak will be. That is mainly because the VCR of the diode is only approximately exponential. Assuming

$$
u=f(i)
$$

Put Equation (2) into Equation (8), do differential both sides of Equation (8) and simplify it.

$$
u=\frac{h_{m}}{k_{m}} f^{\prime}\left(h_{m} \int_{0}^{t} i \mathrm{~d} t\right) i
$$

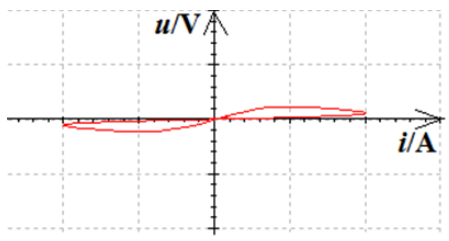

Figure 7. The VCR when $k_{m}=5000$.

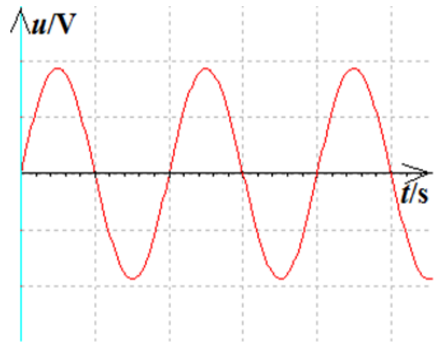

Figure 8. The $u$-t relation when $k_{m}=10^{-7}$.

It can be seen that if VCR is not standard exponential relationship, the equivalent circuit will be affected by $h_{m}$.

Assigning $\mathrm{L}=1 \mathrm{mH}, \mathrm{C}=1 \mathrm{mF}, i=\sin (2 \pi f \mathrm{t}) \mathrm{A}$ and $f=$ $1 \mathrm{kHz}$. When $h_{m}$ is not largely changed, only the voltage peak in the $u$ - $t$ curve increasing, while the shape of the curve does not changing. When $h_{m}$ is bigger than $10^{4}$, the shape of the $u$ - $t$ curve will obviously change, the voltage peak is not being constant, the voltage near the peak varies very quickly and the maximum positive value and the maximum negative value is not equal. Assigning $k_{m}=$ $1000, h_{m}=10^{4}$, the $u$ - $t$ curve of the equivalent circuit is shown in Figure 9. The scales of the horizontal axis and the longitudinal axis are $500 \mathrm{us} / \mathrm{Div}$ and $5 \mathrm{~V} / \mathrm{Div}$ respectively. It can be seen that the $u-t$ relation is no longer periodic.

In the same way, increasing $h_{m}$, the $u$-i curve of the equivalent circuit changes a lot, from a convex and oblique " 8 " changes to a concave and oblique "8", which is quite different from the $u-i$ curve in literature [1]. Figure 10 is the $u$-i curve when $k_{m}=1000, h_{m}=10^{4}$. Both the scales of the horizontal axis and the longitudinal axis are $1 \mathrm{~V} / \mathrm{Div}$. It can be seen that the curve obviously twists and different from the curve in Figure 4. Figure 11 is the amplified curve partially by changing the scale of the horizontal axis to $10 \mathrm{~V} / \mathrm{Div}$ and the longitudinal axis to $50 \mathrm{mV} / \mathrm{Div}$. It can be seen that the $u$ - $i$ curve is composed of many intersected curves. So when $h_{m}=10^{4}$, the equivalent circuit is no longer stable and changes in every period of the current source, which is also why the voltage peak in Figure 10 is a fixed value.

Not only the voltage peak and the $u$ - $t$ relation, but also the stability of circuit will be affected by increasing $h_{m}$. The bigger $h_{m}$, the less stable the circuit. The $u$-i curve of the equivalent circuit gets flatter, approaches a straight line and closer to the horizontal axis when decreasing $h_{m}$. Assigning $k_{m}=1000, h_{m}=0.1$, the simulated VCR is 


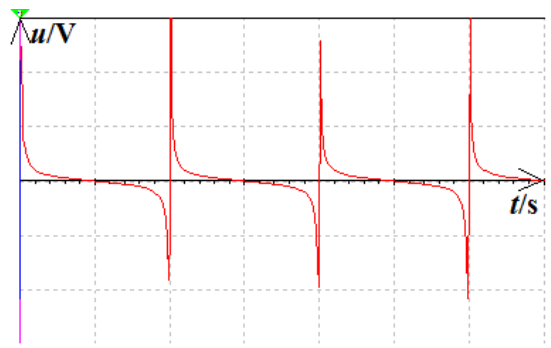

Figure 9. The $u$ - $t$ curve when $h_{m}=10^{4}$.

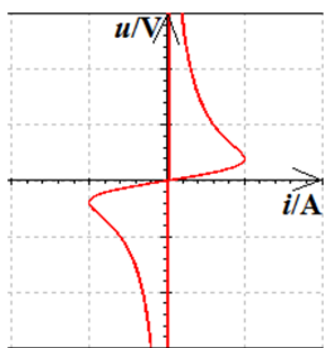

Figure 10. The $u$-i curve when $h_{m}=10^{4}$.

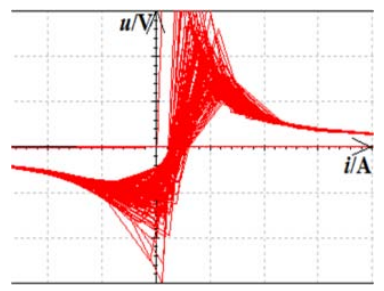

Figure 11. The amplified $u$-i curve when $h_{m}=10^{4}$.

shown in Figure 12. Both the horizontal axis scale and the longitudinal axis scale are $500 \mathrm{mV} / \mathrm{Div}$. The $u$ - $t$ curve is shown in Figure 13, the scales of the horizontal axis and the longitudinal axis are $1 \mathrm{~ms} / \mathrm{Div}$ and $200 \mathrm{mV} / \mathrm{Div}$ respectively. It can be seen that the voltage peak changes less than that of Figure 3. When $h_{m}$ is not largely changed, only the voltage peak of the $u$ - $t$ curve decreases, the shape of the curve doesn't change much and the curve gets smoother. When $h_{m}$ is smaller than 0.01 , the $u$ - $t$ curve significantly changes and closes to the normal sinusoid. Assigning $k_{m}=1000, h_{m}=0.1$, the $u$ - $t$ curve is shown in Figure 14, the horizontal axis scale is 500 us/Div and the longitudinal axis scale is $50 \mathrm{mV} / \mathrm{Div}$. It can be seen that the curve is very close to normal sinusoid, while the $u$-i curve of the equivalent circuit is close to a straight line, the circuit shows the characteristic of linear resistor rather than memristor with changing memrisistance. The stability of the circuit will not be affected when $h_{m}$ decreases. So when $k_{m}=1000, h_{m}=0.01$ $\sim 10^{4}$, the error of the equivalent circuit is small.

\subsection{The Influences of $C$ and $L$}

It can be seen from Equation (7) that the capacitor and inductor have no influences on the equivalent circuit. The

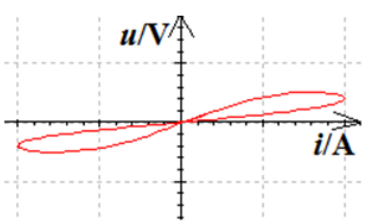

Figure 12. The $u$-i curve when $h_{m}=0.1$.

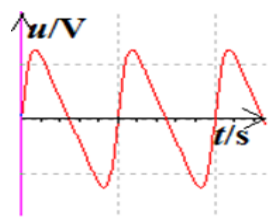

Figure 13. The $u$-t curve when $h_{m}=0.1$.

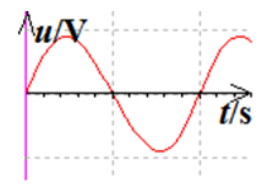

Figure 14. The $u$-t curve when $h_{m}=0.01$.

simulation result is the same with that of the theoretical analysis. Although the capacitance and inductance in the equivalent circuit are adjustable, the curves of VCR and $u-t$ don't change with the capacitance and inductance. When the inductance is in a certain range, assigning $i=$ $\sin (2 \pi f \mathrm{t}) \mathrm{A}, f=1 \mathrm{kHz}, h_{m}=0.4, k_{m}=1000$, changing the capacitance or inductance respectively, the curves of VCR and $u$ - $t$ are totally the same with that of Figure 3 and Figure 4, which shows that the capacitor and inductor have no influences on the equivalent circuit. However, when the inductance is bigger than $1 \mathrm{mH}$, there will be errors in simulation. Though simulation can be continued by cutting down the peak value of current source, the $u-i$ curve is close to a straight line and the $u$ - $t$ curve is close to sinusoid because of the too small current. So the circuit shows the characteristic of linear resistor and cannot represent memristor. Such simulation circumstances won't happen when changing the capacitance.

\subsection{The Influences of the Source's Peak and Frequency}

It can be seen from Equation (7) that the current source's peak has impact on the VCR and $u$ - $t$ relation of the equivalent circuit. Put $i=\operatorname{Isin}(2 \pi f \mathrm{t})$ into Equation (7) and simplify it.

$$
u=\frac{U_{t}}{k_{\mathrm{m}}} \frac{2 \pi f \sin (2 \pi f t)}{\cos (2 \pi f)-1}
$$

It can be seen from Equation (10) that the voltage has no relationship with the current peak. When increasing the current peak, the horizontal peak increases, but the vertical peak doesn't change. So the bigger the current 
peak, the flatter the $u$-i curve, and closer to the horizontal axis.

Assigning $i=\operatorname{Isin}(2 \pi f \mathrm{t}) \mathrm{A}, f=1 \mathrm{kHz}, h_{m}=0.4, k_{m}=$ 1000, $\mathrm{L}=1 \mathrm{mH}, \mathrm{C}=1 \mathrm{mF}$. When $\mathrm{I}=10 \mathrm{~A}$ the $u$-i curve is shown in Figure 15, the scales of the horizontal axis and the longitudinal axis are $5 \mathrm{~V} / \mathrm{Div}$. It can be seen that the curve is very close to the horizontal axis. When the current peak I is bigger than $50 \mathrm{~A}$, the stability of the equivalent circuit weakens and the $u$ - $t$ curve loses periodicity. When I = 50 A the $u$ - $i$ curve is shown in Figure 16, the scales of the horizontal axis and the longitudinal axis are $2 \mathrm{~V} / \mathrm{Div}$. It can be seen that the equivalent circuit is very unstable, many curves lap over, and the analysis result of the circuit is not accurate. When decreasing the current, the $u-i$ curve is close to a straight line. However, the slope $\mathrm{k}$ of the curve keeps constant when changing the current value. This is mainly because that the current value is so small that the resistance of the equivalent circuit varies less and can be approximately regarded as a linear resistor in a short period. For example, when I = 0.01 A the $u$ - $i$ curve is shown in Figure 17. The scales of the horizontal axis and the longitudinal axis are 10 $\mathrm{mV} / \mathrm{Div}$. It can be seen that the $u$-i curve is very close to a straight line. So the circuit shows the characteristic of linear resistor and the characteristic of memristor is not obvious. However, the stability of circuit will not be affected when decreasing the current. In a word, the circuit is not stable if the current is very big, and the circuit will show the characteristic of linear resistor if the current is over-small. So the equivalent circuit in Figure 1 can simulate the memristor only when the effective value of the current is $0.01 \sim 50 \mathrm{~A}$.

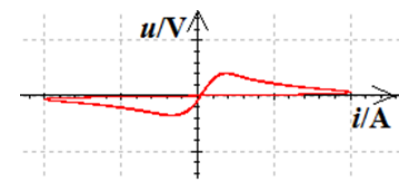

Figure 15. The VCR when $I=10 \mathrm{~A}$.

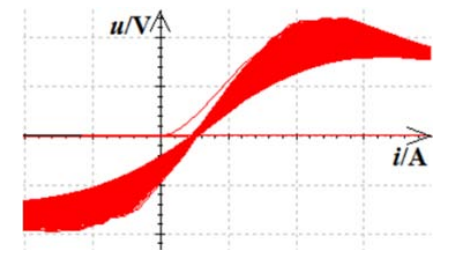

Figure 16. The $u$-i curve when $I=50$ A.

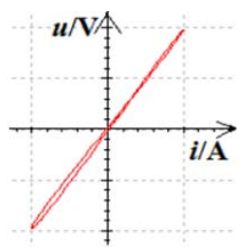

Figure 17. The $u$-i curve when $I=0.01 \mathrm{~A}$.
It can be seen from Equation (10) that the frequency of the current source has impact on the VCR and $u$ - $t$ curve of the equivalent circuit. Assigning $i=\sin (2 \pi f \mathrm{t}) \mathrm{A}, h_{m}=$ $0.4, k_{m}=1000, \mathrm{~L}=1 \mathrm{mH}, \mathrm{C}=1 \mathrm{mF}$. Increasing the frequency of current source in a certain range, the $u$ - $t$ curve doesn't change obviously, and the node of the oblique " 8 " of the $u$-i curve offsets upward but not so obviously.

Assigning $f=100 \mathrm{kHz}$, the $u-i$ curve of the equivalent circuit is shown in Figure 18. The scales of the horizontal axis and the longitudinal axis are $500 \mathrm{mV} / \mathrm{Div}$. It can be seen that the $u$ - $i$ curve is no longer an oblique " 8 " but an oval, the circuit is unstable, and the curve is composed of many curves. The $u$ - $t$ curve of the equivalent circuit is shown in Figure 19. The scales of the horizontal axis and the longitudinal axis are $10 \mathrm{us} / \mathrm{Div}$ and $500 \mathrm{mV} / \mathrm{Div}$ respectively. It can be seen that the curve is close to sinusoid, the voltage peak is bigger in the first cycle of the current source, and then the voltage peak gets to stable, so the stability of circuit gets less. When decreasing the frequency of the current source, the $u$-i curve gets flatter and closes to the horizontal axis, and the characteristic of the equivalent circuit approaches to the linear resistor. The $u$ - $t$ curve has not obviously change. Assigning $\mathrm{f}=$ $100 \mathrm{~Hz}$, the $u$ - $i$ curve of the equivalent circuit is shown in Figure 20. The scales of the horizontal axis and the longitudinal axis are $500 \mathrm{mV} / \mathrm{Div}$. It can be seen that the curve is very similar to Figure 16. In a word, if the frequency is over high, the $u$ - $i$ curve of the equivalent circuit is obviously distorted and the circuit is unstable, and

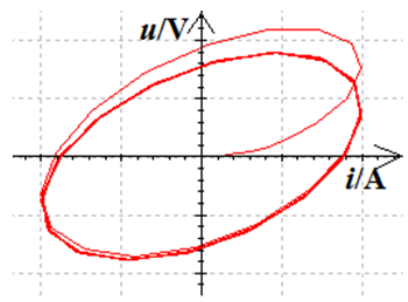

Figure 18. The $u$-i curve when $f=100 \mathrm{kHz}$.

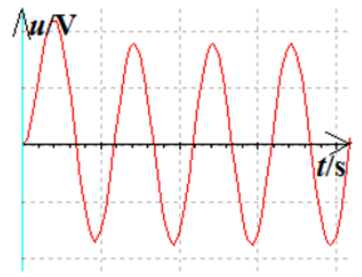

Figure 19. The $u$-t curve when $f=100 \mathrm{kHz}$.

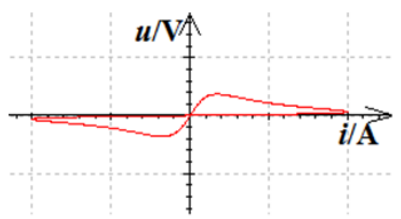

Figure 20. The $u$-i curve when $f=100 \mathrm{~Hz}$. 
if the frequency is over small, the circuit will show the characteristic of linear resistor. Thus the equivalent circuit in Figure 1 can simulate the memorize only when the frequency of the current source is $0.1 \sim 10 \mathrm{kHz}$.

\section{Conclusion}

A mutator is built based on Multisim as an equivalent circuit of memristor in this paper. The parameters which impact on the equivalent circuit are analyzed, and the working conditions of the equivalent circuit of memristor are given. The errors of the equivalent circuit are smaller when $k_{m}$ is $10 \sim 10^{4}$ and $h_{m}$ is $0.01 \sim 10^{4}$, the inductor and capacitor have no effects on the equivalent circuit when $\mathrm{L}<1 \mathrm{mH}$, the frequency should be $f<10 \mathrm{kHz}$, and the current should be I $<50$ A. Which means the circuit can not be used in high frequency circuit.

\section{Acknowledgements}

The work is supported by the Research Innovation Fund for College Students of Beijing University of Posts and Telecommunications.

\section{REFERENCES}

[1] D. B. Strukov, G. S. Snider, D. R. Stewart and R. S.Williams, "The Missing Memristor Found," Nature, Vol. 453, 2008, pp. 80-83.

[2] X. Zhang, Y. Z. Zhou, Q. Bi, X. H. Yang and Y. X. Zu, "The Mathematical Model and Properties of Memristor with Border Constraint," Acta Physica Sinica, Vol. 59, No. 9, 2010, pp. 6669-6676.

[3] Q. H. Wang and W. P. Song, "Realization of Memristor by Two-Port Mutator," Journal of Electrical \& Electronic Education, Vol. 33, No. 3, 2011, pp. 56-57.
[4] M. Itoh and L. O. Chua, "Memristor Oscillators,” International Journal of Bifurcation and Chaos, Vol. 18, No. 11, 2008, pp. 3183-3206. http://dx.doi.org/10.1142/S0218127408022354

[5] Y. Zhang, X. L. Zhang and J. B. Yu, "Approximated SPICE Model for Emristor," International Conference on Communications, Circuits and Systems, 2009, pp. 928931. http://dx.doi.org/10.1109/ICCCAS.2009.5250371

[6] D. P. Wang, Z. H. Hu, X. Yu and J. B. Yu, "A PWL Model of Memristor and Its Application Example," International Conference on Communications, Circuits and Systems, 2009, pp. 932-934, http://dx.doi.org/10.1109/ICCCAS.2009.5250372

[7] Klaus Witrisal, "A Memristor-Based Multicarrier UWB Receiver,” Proceedings of IEEE International Conference on Ultra-Wideband, IEEE Press, Sep. 2009, pp. 678-683, http://dx.doi.org/10.1109/ICUWB.2009.5288703

[8] D. Varghese and G. Gandhi, "Memristor Based High Linear Range Differential Pair," International Conference on Communications, Circuits and Systems, Jul. 2009, pp. 935-938. http://dx.doi.org/10.1109/ICCCAS.2009.5250373

[9] A. Delgado, "Input-Output Linearization of Memristive Systems," Proceedings of IEEE Nanotechnology Materials and Devices Conference, IEEE Press, July. 2009, pp. 154-157. http://dx.doi.org/10.1109/NMDC.2009.5167523

[10] M. Di Ventura, Y. V. Pershin and L. O. Chua, "Circuit Elements with Memory: Memristors, Memcapacitors, and Meminductors," Proceedings of IEEE, Vol. 97, Oct. 2009, pp. 1717-1724. http://dx.doi.org/10.1109/JPROC.2009.2021077

[11] L. O. Chua, "Memristor-The Missing Circuit Element," IEEE Transactions on Circuit Theory, Vol. 18, Sep. 1971, pp. 507-519. http://dx.doi.org/10.1109/TCT.1971.1083337 